\title{
Spherical Tikhonov Regularization Wavelets in Satellite Gravity Gradiometry with Random Noise
}

\author{
W. Freeden ${ }^{1}$, S. Pereverzev ${ }^{2}$ \\ ${ }^{1}$ University of Kaiserslautern \\ ${ }^{2}$ Kiev Institute of Mathematics \\ Geomathematics Group \\ Tereshenkivska 3 \\ 67653 Kaiserslautern \\ $252601 \mathrm{Kiev}-4$ \\ Germany \\ Ukraine \\ email: freeden@mathematik.uni-kl.de \\ email: serg-p@mail.kar.net
}

\begin{abstract}
This paper considers a special class of regularization methods for satellite gravity gradiometry based on Tikhonov spherical regularization wavelets with particular emphasis on the case of data blurred by random noise. A convergence rate is proved for the regularized solution, and a method is discussed for choosing the regularization level a posteriori from the gradiometer data.
\end{abstract}

Key words. Ill-posed problem, regularization wavelets, random noise, rate of convergence, satellite gravity gradiometry.

Correspondence to W. Freeden 


\section{Introduction}

The primary aim of the planned GOCE-Mission (cf. ESA (1999)) is to provide a unique model of the earth's gravitational field and of its equipotential reference surface of the geoid on a global scale with high-spatial-resolution. Such an advance in the knowledge of the earth's gravitational field will help to develop a much deeper understanding of how the earth's interior system works. Fundamental insight is expected into a wide range of research and application areas, including solid earth physics, oceanography, and physical geodesy. To reach the measurement goal and to meet the scientific objectives, the payload consists of an electrostatic gradiometer and a combined GPS/GLONASS precise positioning system. (For more details about satellite gravity gradiometry (SGG) the reader is referred e.g. to ESA (1999), FREEdEN (1999), ILK (1993), JEKELI (1988), Kusche (1998), RuMmel (1997), Rummel et al. (1993), Schneider (1997), Tscherning et al. (1990) and the references therein).

The GOCE-orbit is quite attractive for mathematical modelling. It is selected in such a way that some simplifying hypotheses may be assumed to be satisfied: (i) a (near) polar orbit allows that no holes are in the data, (ii) a (near) circular orbit implies that the data are lying on a sphere, (iii) the measurements (achieved by employing the principle of gradiometry for the first time in a satellite) provide global information about the second order partial derivatives of the gravitational potential at a moderate satellite altitude.

Of particular interest from mathematical point of view is the use of second order radial derivatives, which indeed can be chosen to guarantee the uniqueness of the gravitational potential (for more details establishing uniqueness results in satellite gravity gradiometry from orbital components of the Hesse matrix the reader is referred to FREEDEN et al. (1997), FREEDEN (1999)).

Using second order radial derivatives on the orbital sphere the spherical framework of satellite gravity gradiometry can be mathematically formulated in the form of the first kind integral equation

$$
\Lambda_{\frac{R}{r}} F(x):=\int_{\Omega_{R}} K_{\Lambda \frac{R}{r}}(x, y) F(y) d \omega_{R}(y)=G(x), \quad x \in \Omega_{r},
$$

where $\Omega_{R}, \Omega_{r}$ are the spherical surfaces of the earth and the satellite orbit, 
respectively, and the kernel of $\Lambda_{\frac{R}{r}}$ is given by

$$
K_{\Lambda_{\frac{R}{r}}}(x, y)=\left(\frac{\partial}{\partial r}\right)^{2} \frac{1}{4 \pi R} \frac{r^{2}-R^{2}}{\left(r^{2}+R^{2}-2 x \cdot y\right)^{3 / 2}}, \quad x \in \Omega_{r}, y \in \Omega_{R} .
$$

A straightforward calculation (see e.g. FREEDEN et al. (1997)) shows that we can rewrite $\Lambda_{\frac{R}{r}}$ in the form of a singular-value decomposition

$$
\Lambda_{\frac{R}{r}}=\sum_{n=0}^{\infty} \sigma_{n} \sum_{k=1}^{2 n+1} Y_{n, k}^{r}(x) \otimes Y_{n, k}^{R}(y),
$$

where

$$
\begin{aligned}
Y_{n, k}^{r}(x) & =\frac{1}{r} Y_{n, k}\left(\frac{x}{r}\right), \quad x \in \Omega_{r}, \\
Y_{n, k}^{R}(y) & =\frac{1}{R} Y_{n, k}\left(\frac{y}{R}\right), \quad y \in \Omega_{R},
\end{aligned}
$$

$\left\{Y_{n, k}\right\}$ is a system of spherical harmoncs $\mathcal{L}_{2}$-orthonormalized with respect to the unit sphere in $\mathbb{R}^{3}$, and $\left\{\sigma_{n}\right\}$ is the symbol of the satellite gravity operator $\Lambda_{\frac{R}{r}}$ given by

$$
\sigma_{n}=\left(\Lambda_{\frac{R}{r}}\right)^{\wedge}(n)=\left(\frac{R}{r}\right)^{n} \frac{(n+1)(n+2)}{r^{2}}, \quad n=0,1, \ldots
$$

Consequently,

$$
\ln \sigma_{n}=-n \ln \frac{r}{R}+O(\ln n), \quad n \rightarrow \infty .
$$

It means that $\Lambda_{\frac{R}{r}}$ is a compact operator. Remembering Hadamard's definition of a well-posed problem (existence, uniqueness, continuity of the inverse), we consequently see that the problem (1) is ill-posed as it violates the third condition due to the compactness of $\Lambda_{\frac{R}{r}}$. Moreover, since $\sigma_{n}^{-1}$ diverges exponentially fast for $n \rightarrow \infty$, the inverse problem given by (1) is classified to be exponentially or severely ill-posed (see e.g. LouIs (1982)), and the crux of the difficulty is that usually in practice we are confronted with satellite data blurred by random noise. In other words, we are interested in the solution of the inverse problem

$$
\Lambda_{\frac{R}{r}} F=G^{\varepsilon}
$$


with perturbed right hand side given by

$$
G^{\varepsilon}=G+\varepsilon \xi
$$

where $\varepsilon$ is a small positive number, used for measuring the noise level, and $\xi$ is some zero-mean stochastic process with the covariance operator

$$
\operatorname{cov}(\xi)=I
$$

( $I$ is the identity operator). Another way of putting it is that $\xi$ is a weak or generalized random element such that for any element $g$ from the space $\mathcal{L}^{2}\left(\Omega_{r}\right)$ of square-integrable functions on the sphere $\Omega_{r}$ the inner product $(g, \xi)$ is a measurable function, mapping some probability space $(\Sigma, \mathcal{A}, \mathbb{P})$ into $\mathbb{R}$ equipped with its Borel $\sigma$-field. Moreover, for any $f, g \in \mathcal{L}^{2}\left(\Omega_{r}\right)$, the functions $(f, \xi),(g, \xi)$ are square-summable with respect to the probability measure $\mathbb{P}$ and

$$
\mathbb{E}(f, \xi)=\mathbb{E}(g, \xi)=0, \quad \mathbb{E}\{(f, \xi) \cdot(g, \xi)\}=(f, g),
$$

where $\mathbb{E}$ is the expectation with respect to $\mathbb{P}$. Note that our assumptions as to the random noise $\xi$ are rather general, because we don't impose any restrictions on the probability distribution of it (e.g. to be Gaussian noise and the like).

The inverse problem (1) with random noise is the problem of statistical estimation of the unknown solution $F=F_{*}$ based on the observation $G^{\varepsilon}$. The study of such problems is in the focus of the recent statistical literature (Korostelev, Tsybakov (1993), Nussbaum (1994), Donoho (1995), Mair, RuymgaArt (1996), Golubev, Khasiminskil (1997)), to mention a few, and typically some finite linear combination of the elements from the singular value decomposition of the operator involved in the inverse problem plays the role of the estimator. So, in our case such an estimator would be a bandlimited function or a function having a finite number of non-zero spherical harmonic coefficients. But this approach has some disadvantage if only a local data set is available, since local structures are not reflected in the spherical harmonic coefficients. This disadvantage can be overcome by using recently developed spherical regularization wavelet theory (FreEden et al. (1998), FreEden, SchneIDER (1998)), because non-bandlimited spherical regularization wavelets packets are nearly locally supported and appropriate wavelet coefficients have a strong connection to the corresponding local region. It should be remarked that the idea to use wavelet-based regularization techniques for inverse estimation in the presence of random noise has already been reported in DoNOHO (1995), but it 
has been essentially used there that the considered inverse problem has a finite degree of ill-posedness or, what is the same, is not severely ill-posed. It is the purpose of this paper to obtain an error estimate for non-bandlimited spherical regularization wavelets in the severely ill-posed gradiometry problem (1) with random noise. Note that for deterministic noise in the bandlimited case it has been done recently in Pereverzev, Schock $(1999,2000)$. Moreover, attention will be centered on the spherical regularization wavelet packets corresponding to the simplest version of Tikhonov regularization. There are at least two reasons for it. Namely, such wavelets possess the excellent space localization properties (cf. SCHNEIDER (1997)), and moreover, as it has been shown recently in PeREVERzEv, SCHOCK (2000), the simplest Tikhonov regularization can insure the best possible order of accuracy (for deterministic noise) without any information about the smoothness of the unknown solution.

\section{Spherical Tikhonov regularization wavelets}

Spherical Tikhonov regularization wavelets are based on the Tikhonov regularization of the inverse problem (4). In accordance with the approach Freeden et al. (1998), Freeden, Schneider (1998) the Tikhonov regularized solution $F_{\gamma}^{\varepsilon}$ is obtained by decomposition and reconstruction of the noisy righthand side $G^{\varepsilon}$ with respect to the wavelet basis which consists of rotated and dilated copies of a mother wavelet.

Let $\left\{\gamma_{j}\right\}_{j=0}^{\infty}$ be a strictly decreasing sequence of real numbers satisfying $\lim _{j \rightarrow \infty} \gamma_{j}=0$. Recall that Tikhonov regularized solution $F_{j}^{\varepsilon}=F_{\gamma_{j}}^{\varepsilon}$ of (4) corresponding to the regularization parameter $\gamma=\gamma_{j}$ is defined by

$$
F_{j}^{\varepsilon}=\left(\gamma_{j}^{2} I+\Lambda_{\frac{R}{r}}^{*} \Lambda_{\frac{R}{r}}\right)^{-1} \Lambda_{\frac{R}{r}}^{*} G^{\varepsilon} .
$$

Using singular-value decomposition (2) we can represent it as follows

$$
F_{j}^{\varepsilon}(x)=\int_{\Omega_{r}} S_{j}(x, y) G^{\varepsilon}(y) d \omega_{r}(y), \quad x \in \Omega_{R}
$$

where

$$
S_{j}(x, y)=\sum_{n=0}^{\infty} \sum_{k=1}^{2 n+1} \frac{\sigma_{n}}{\sigma_{n}^{2}+\gamma_{j}^{2}} Y_{n, k}^{R}(x) Y_{n, k}^{r}(y) .
$$

Now we are in a position to apply the addition theorem

$$
\sum_{k=1}^{2 n+1} Y_{n, k}^{R}(x) Y_{n, k}^{r}(y)=\frac{2 n+1}{4 \pi R r} P_{n}\left(\frac{x \cdot y}{R r}\right), \quad x \in \Omega_{R}, y \in \Omega_{r},
$$


and to reduce the kernel $S_{j}(x, y)$ to a so-called radial basis function which only depends on the inner product $x \cdot y$ of $x \in \Omega_{R}$ and $y \in \Omega_{r}$

$$
S_{j}(x, y)=\sum_{n=0}^{\infty} \frac{2 n+1}{4 \pi R r} \frac{\sigma_{n}}{\sigma_{n}^{2}+\gamma_{j}^{2}} P_{n}\left(\frac{x \cdot y}{R r}\right),
$$

where $P_{n}$ is the Legendre polynomial of degree $n$. In order to proceed in Tikhonov spherical regularization wavelets we introduce the spherical scaling function

$$
\Phi_{j}(t)=\sum_{n=0}^{\infty} \frac{2 n+1}{4 \pi} \hat{\Phi}_{j}(n) P_{n}(t), \quad t \in[-1,+1],
$$

with

$$
\hat{\Phi}_{j}(n)=\frac{\sigma_{n}}{\sigma_{n}^{2}+\gamma_{j}^{2}}
$$

corresponding to the Tikhonov regularization method. Moreover, we call $\left\{\Psi_{j}(t)\right\}_{j=0}^{\infty}$, defined by

$$
\Psi_{j}(t)=\sum_{n=0}^{\infty} \frac{2 n+1}{4 \pi} \hat{\Psi}_{j}(n) P_{n}(t), \quad t \in[-1,+1]
$$

with

$$
\hat{\Psi}_{j}(n)=\left[\left(\hat{\Phi}_{j+1}(n)\right)^{2}-\left(\hat{\Phi}_{j}(n)\right)^{2}\right]^{1 / 2},
$$

the scale discrete Tikhonov spherical regularization wavelet packet. As in FrEeden et al. (1997), FreEDEN, SchNEIDER (1998) we define the dilation operators $D_{p}, p=0,1, \ldots$, acting on the families $\left\{\Phi_{j}\right\},\left\{\Psi_{j}\right\}$ in the following way: $D_{p} \Phi_{j}=\Phi_{j+p}, D_{p} \Psi_{j}=\Psi_{j+p}$. In particular, we obtain $\Psi_{j}=D_{j} \Psi_{0}$. Thus, we refer to $\Psi_{0}$ and $\Phi_{0}$ as the 'mother wavelet packet', and 'mother scaling function', respectively. Moreover, we define a rotation (shifting) operator $R_{z}^{r}$ on $\Omega_{r}$ by

$$
\begin{aligned}
R_{z}^{r} \Phi_{j}(x)=R_{z}^{r} D_{j} \Phi_{0}(x) & =\sum_{n=0}^{\infty} \sum_{k=1}^{2 n+1} \hat{\Phi}_{j}(n) Y_{n, k}^{R}(x) Y_{n, k}^{r}(z) \\
& =\sum_{n=0}^{\infty} \hat{\Phi}_{j}(n) \frac{2 n+1}{4 \pi R r} P_{n}\left(\frac{x \cdot z}{R r}\right)
\end{aligned}
$$

Certainly, all choices of $r$ are possible in (8), especially the case $r=R$. In the same manner we define $R_{z}^{r} D_{j} \Psi_{0}(x)$, which can be interpreted as a 
dilated and rotated copy of the 'mother wavelet' $\Psi_{0}$. Now using Theorem 4.6 in FREEDEN, SCHNEIDER (1998) we can represent the Tikhonov regularized solution $F_{m}^{\varepsilon}$ as follows:

$$
\begin{aligned}
F_{m}^{\varepsilon}(x) & =\int_{\Omega_{R}}\left(\int_{\Omega_{r}} R_{z}^{r} \Phi_{0}(y) G^{\varepsilon}(y) d \omega_{r}(y)\right) R_{z}^{R} \Phi_{0}(x) d \omega_{R}(z) \\
& +\sum_{j=0}^{m} \int\left(\int_{\Omega_{R}} R_{z}^{r} D_{j} \Psi_{0}(y) G^{\varepsilon}(y) d \omega_{r}(y)\right) R_{z}^{R} D_{j} \Psi_{0}(x) d \omega_{R}(z) .
\end{aligned}
$$

This formula enables us to pass over from one regularized solution $F_{m}^{\varepsilon}$ to another $F_{m+1}^{\varepsilon}$ by adding the so-called detail information of level $m$ as the difference of two smoothings at two consecutive scales $m+1$ and $m$ (see (7)).

A very important question is the relation between the regularization level $m$ and the noise level $\varepsilon$. This question is discussed in the next sections in more detail.

\section{An error estimate}

As it is usual in statistics, the performance of some estimator $F^{\varepsilon}(\xi)$ of the unknown solution $F_{*}$ from indirect measurements (4), blurred by random noise $\xi$ will be based on the risk $\mathbb{E}\left\|F_{*}-F^{\varepsilon}(\xi)\right\|_{\mathcal{L}^{2}\left(\Omega_{R}\right)}^{2}$. For the spherical Tikhonov regularization wavelet estimator $F_{J}^{\varepsilon}$, making use of (2), (5), (6) and the notation

$$
T_{J}=\left(\gamma_{J}^{2} I+\Lambda_{\frac{R}{r}}^{*} \Lambda_{\frac{R}{r}}\right)^{-1} \Lambda_{\frac{R}{r}}^{*}
$$

this can be written as

$$
\begin{aligned}
\mathbb{E}\left\|F_{*}-F_{J}^{\varepsilon}\right\|_{\mathcal{L}_{2}\left(\Omega_{R}\right)}^{2} & =\mathbb{E}\left\|\left(I-T_{J} \Lambda_{\frac{R}{r}}\right) F_{*}+\varepsilon T_{J} \xi\right\|_{\mathcal{L}_{2}\left(\Omega_{R}\right)}^{2} \\
& =\mathbb{E}\left\|\left(I-T_{J} \Lambda_{\frac{R}{r}}\right) F_{*}\right\|_{\mathcal{L}_{2}\left(\Omega_{R}\right)}^{2}+\varepsilon^{2} \mathbb{E}\left\|T_{J} \xi\right\|_{\mathcal{L}_{2}\left(\Omega_{R}\right)}^{2} \\
& -2 \varepsilon \mathbb{E}\left(T_{J}^{*}\left(I-T_{J} \Lambda_{\frac{R}{r}}\right) F_{*}, \xi\right) \\
& =\left\|\left(I-T_{J} \Lambda_{\frac{R}{r}}\right) F_{*}\right\|_{\mathcal{L}_{2}\left(\Omega_{R}\right)}^{2}+\varepsilon^{2} \mathbb{E}\left\|T_{J} \xi\right\|_{\mathcal{L}_{2}\left(\Omega_{R}\right)}^{2}
\end{aligned}
$$

The last two terms in (9) are treated in statistics as bias and variance of the risk, respectively. Now we turn to estimate these quantities separately. 
Using a singular-value decomposition (2) we have

$$
\begin{aligned}
\left\|T_{J} \xi\right\|_{\mathcal{L}_{2}\left(\Omega_{R}\right)}^{2} & =\left\|\left(\gamma_{J}^{2} I+\Lambda_{\frac{R}{r}}^{*} \Lambda_{\frac{R}{r}}\right)^{-1} \Lambda_{\frac{R}{r}}^{*} \xi\right\|_{\mathcal{L}_{2}\left(\Omega_{R}\right)}^{2} \\
& =\sum_{n=0}^{\infty}\left(\frac{\sigma_{n}}{\gamma_{J}^{2}+\sigma_{n}^{2}}\right)^{2} \sum_{k=1}^{2 n+1}\left(Y_{n, k}^{r}, \xi\right)^{2} .
\end{aligned}
$$

Now from (3) and (5) it follows that for any $\beta>1$

$$
\begin{aligned}
\mathbb{E}\left\|T_{J} \xi\right\|_{\mathcal{L}_{2}\left(\Omega_{R}\right)}^{2} & =\sum_{n=0}^{\infty}\left(\frac{\sigma_{n}}{\gamma_{J}^{2}+\sigma_{n}^{2}}\right)^{2} \sum_{k=1}^{2 n+1} \mathbb{E}\left(Y_{n, k}^{r}, \xi\right)^{2} \\
& =\sum_{n=0}^{\infty}\left(\frac{\sigma_{n}}{\gamma_{J}^{2}+\sigma_{n}^{2}}\right)^{2}(2 n+1) \\
& =\sum_{n=0}^{\infty}\left[\frac{\sigma_{n} \ln ^{\beta} \sigma_{n}^{-1}}{\gamma_{J}^{2}+\sigma_{n}^{2}}\right]^{2} \ln ^{-2 \beta} \sigma_{n}^{-1}(2 n+1) \\
& \leq C \sum_{n=0}^{\infty}\left[\frac{\sigma_{n} \ln ^{\beta} \sigma_{n}^{-1}}{\gamma_{J}^{2}+\sigma_{n}^{2}}\right]^{2} n^{-2 \beta+1} \\
& \leq C\left[\max _{0 \leq \lambda \leq 1}\left|g_{\beta}(\lambda)\right|\right]^{2}
\end{aligned}
$$

where $C$ depends only on $R, r, \beta$, and $g_{\beta}$ is given by

$$
g_{\beta}(\lambda):=\frac{\lambda \ln ^{\beta} \frac{1}{\lambda}}{\gamma_{J}^{2}+\lambda^{2}}
$$

It is easy to see that

$$
g_{\beta}^{\prime}(\lambda)=-u(\lambda)\left(\gamma_{J}^{2}+\lambda^{2}\right)^{-2} \ln ^{\beta-1} \frac{1}{\lambda}
$$

where

$$
u(\lambda)=\left(\lambda^{2} \ln \frac{1}{\lambda}+\beta \lambda^{2}-\gamma_{J}^{2} \ln \frac{1}{\lambda}+\beta \gamma_{J}^{2}\right) .
$$

Keeping in mind that, for $\lambda \in(0,1]$,

$$
u^{\prime}(\lambda)=2 \lambda \ln \frac{1}{\lambda}+(2 \beta-1) \lambda+\lambda^{-1} \gamma_{J}^{2}>0
$$

and $\lim _{\lambda \rightarrow 0} u(\lambda)=-\infty$, but $u(1)=\beta\left(1+\gamma_{J}^{2}\right)>0$, we conclude that there is only one $\lambda_{0} \in[0,1]$ such that $u\left(\lambda_{0}\right)=0$ and for all $\lambda \in[0,1], g_{\beta}(\lambda) \leq g_{\beta}\left(\lambda_{0}\right)$. 
Let now $\kappa \in(0,1)$ be such that $\beta\left(1+\kappa^{2}\right)\left(1-\kappa^{2}\right)^{-1}=\ln \frac{1}{\kappa}$ (it is easy to check that such $\kappa$ exists). Then $\lambda_{0}>\kappa \gamma_{J}$ because $u(\lambda)$ monotonically increases and

$$
\begin{aligned}
u\left(\kappa \gamma_{J}\right) & =\gamma_{j}^{2}\left[\left(\kappa^{2}-1\right) \ln \frac{1}{\kappa \gamma_{J}}+\beta\left(1+\kappa^{2}\right)\right] \\
& <\gamma_{J}^{2}\left[\left(\kappa^{2}-1\right) \ln \frac{1}{\kappa}+\beta\left(1+\kappa^{2}\right)\right] \\
& =0=u\left(\lambda_{0}\right) .
\end{aligned}
$$

Moreover, $\lambda_{0}<\gamma_{J}$ because of $u\left(\lambda_{0}\right)=0<2 \beta \gamma_{J}^{2}=u\left(\gamma_{J}\right)$. Thus,

$$
\begin{aligned}
g_{\beta}\left(\lambda_{0}\right) & =\frac{\lambda_{0} \ln ^{\beta} \frac{1}{\lambda_{0}}}{\gamma_{J}^{2}+\lambda_{0}^{2}} \leq \frac{\lambda_{0} \ln ^{\beta} \frac{1}{\lambda_{0}}}{\gamma_{J}^{2}} \\
& \leq \gamma_{J}^{-1} \ln ^{\beta} \frac{1}{\lambda_{0}} \leq \gamma_{J}^{-1} \ln ^{\beta} \frac{1}{\kappa \gamma_{J}} \\
& \leq C \gamma_{J}^{-1} \ln ^{\beta} \frac{1}{\gamma_{J}},
\end{aligned}
$$

and

$$
\begin{aligned}
\mathbb{E} & \left\|T_{J} \xi\right\|_{\mathcal{L}_{2}\left(\Omega_{R}\right)}^{2} \\
& \leq C\left[\max _{0 \leq \lambda \leq 1}\left|g_{\beta}(\lambda)\right|\right]^{2} \leq C\left[g_{\beta}\left(\lambda_{0}\right)\right]^{2} \leq C \gamma_{J}^{-2} \ln ^{2 \beta} \frac{1}{\gamma_{J}} .
\end{aligned}
$$

To estimate the bias we consider the frequently used constrain of assuming that the exact solution $F_{*}$ of the equation (1) belongs to the spherical Sobolev space $\mathcal{H}_{s}\left(\Omega_{R}\right)$ for some $s>0$. The scale of the spherical Sobolev spaces has been introduced in FREEDEN et al. (1998), and $\mathcal{H}_{s}\left(\Omega_{R}\right)$ is the completion of the space

$$
\left\{H: H \in \mathcal{L}_{2}\left(\Omega_{R}\right), \quad\|H\|_{s}^{2}:=\sum_{n=0}^{\infty} \sum_{k=1}^{2 n+1}\left(n+\frac{1}{2}\right)^{2 s}\left(Y_{n, k}^{R}, H\right)^{2}<\infty\right\}
$$

under the norm $\|\cdot\|_{s}$. Motivated by the arguments of Lemma 1 and Lemma 3 by Pereverzev, Schock (2000) we are led to the following: We immediately see that for $F_{*} \in \mathcal{H}_{s}\left(\Omega_{R}\right)$

$$
\begin{aligned}
\left\|F_{*}-T_{J} \Lambda_{\frac{R}{r}} F_{*}\right\|_{\mathcal{L}^{2}\left(\Omega_{R}\right)}^{2} & \\
& =\sum_{n=0}^{\infty}\left(\frac{\gamma_{J}^{2}}{\gamma_{J}^{2}+\sigma_{n}^{2}}\right)^{2}\left(n+\frac{1}{2}\right)^{-2 s} \sum_{k=1}^{2 n+1}\left(Y_{n, k}^{R}, F_{*}\right)^{2}\left(n+\frac{1}{2}\right)^{2 s} .
\end{aligned}
$$


In connection with (3), i.e. $n=\ln ^{-1} \frac{r}{R} \ln \frac{1}{\sigma_{n}}+O(\ln n)$, and the discussion of the function $h(\lambda)=\lambda^{-2} \ln ^{-s} \frac{1}{\lambda}, \lambda \in\left(0,\left\|\Lambda_{\frac{R}{r}}\right\|\right.$ ), (observe that $\left\|\Lambda_{\frac{R}{r}}\right\|=$ $\max _{n \in \mathbb{N}_{0}}\left|\left(\Lambda_{\frac{R}{r}}\right)^{\wedge}(n)\right|$, see FrEEDEN (1999)) we find

$$
\left\|F_{*}-T_{J} \Lambda_{\frac{R}{r}} F_{*}\right\|_{\mathcal{L}^{2}\left(\Omega_{R}\right)}=\left\|F_{*}\right\|_{s} \ln ^{s} \frac{r}{R}\left(\ln ^{-s} \frac{1}{\gamma_{J}}+O\left(\gamma_{J}^{2}\right)\right) .
$$

Letting formally $\gamma_{J}=\varepsilon^{1 / 2}$ the right hand side of (11) may be rewritten by $\left\|F_{*}\right\|_{s} 2^{s} \ln ^{s} \frac{r}{R}\left(\ln ^{-s} \frac{1}{\varepsilon}+O(\varepsilon)\right)$. More accurately, the statement of the next theorem follows immediately from (10) and (11).

THEOREM 3.1.If the solution $F_{*}$ of (1) belongs to $\mathcal{H}_{s}\left(\Omega_{R}\right)$ for some $s>0$ then for sufficiently small $\varepsilon$ and $\gamma_{J} \in\left[\varepsilon^{p}, \varepsilon^{\frac{1}{2}}\right], \frac{1}{2}<p<1$,

$$
\left\{\mathbb{E}\left\|F_{*}-F_{J}^{\varepsilon}\right\|_{\mathcal{L}_{2}\left(\Omega_{R}\right)}^{2}\right\}^{1 / 2} \leq C \ln ^{-s} \frac{1}{\varepsilon}
$$

where $C$ depends only on $F_{*}$. In particular,

$$
C=\left\|F_{*}\right\|_{s} \ln ^{s}\left(\frac{r}{R}\right)^{2}(1+o(1)) .
$$

\section{Final Discussion}

First of all we note that for known smoothness index $s$ the same arguments as in GolUBEV, KHASIMINSKII (1997) allow to show that the order of accuracy $O\left(\ln ^{-s} \frac{1}{\varepsilon}\right)$ indicated in our theorem cannot be improved at least for the case of Gaussian white noise $\xi$. On the other hand, Theorem 3.1 shows that this order can be achieved for arbitrary $\gamma_{J} \in\left[\varepsilon^{p}, \varepsilon^{1 / 2}\right], \frac{1}{2}<p<1$. The same situation also takes place for the severely ill-posed problems with deterministic noise $\xi,\|\xi\| \leq 1$, and it explains, in particular, the fact mentioned in MAIR (1994), when the order of accuracy $O\left(\ln ^{-s} \frac{1}{\varepsilon}\right)$ has been achieved for two values of regularization parameter MAIR (1994) and BAUMEISTER (1987) having different orders with respect to $\varepsilon$. But our theorem describes only an asymptotic behaviour of the accuracy for $\varepsilon \rightarrow 0$. For fixed noise level $\varepsilon$ the results of several test calculations presented in SCHNEIDER (1997) demonstrate that Tikhonov spherical regularization wavelet method is rather sensitive to the choise of $\gamma_{J}$. Therefore, for unknown smoothness index $s$ there is a reason to apply some a posteriori parameter choice strategy. For severely ill-posed problems with deterministic noise it has been shown in Pereverzev, Schock (2000) that the combination of Morozov's discrepancy principle with the simplest Tikhonov regularization allow to obtain the 
accuracy of order $O\left(\ln ^{-s} \frac{1}{\varepsilon}\right)$ even if $s$ is unknown. In our case, the discrepancy principle leads to the choice of $\gamma_{J}$ with

$$
J=\min \left\{m:\left\|\Lambda_{\frac{R}{r}} F_{m}^{\varepsilon}-G^{\varepsilon}\right\|_{\mathcal{L}^{2}\left(\Omega_{R}\right)} \leq d \varepsilon, m=1,2, \ldots\right\},
$$

where $d>1$. In WAHBA (1977) it was noted that for the random noise such a form of the discrepancy principle would be 'probably reasonable', too. On the other hand, within the framework of our assumptions (5)

$$
\mathbb{E}\left\|G^{\varepsilon}\right\|_{\mathcal{L}_{2}\left(\Omega_{R}\right)}^{2}=\|G\|_{\mathcal{L}_{2}\left(\Omega_{R}\right)}^{2}+\varepsilon^{2} \mathbb{E}\|\xi\|_{\mathcal{L}_{2}\left(\Omega_{R}\right)}^{2} \geq \varepsilon^{2} \mathbb{E}\|\xi\|_{\mathcal{L}_{2}\left(\Omega_{R}\right)}^{2}=\infty
$$

and in general we cannot expect that the norm of the residuals $\left\|\Lambda_{\underline{R}} F_{m}^{\varepsilon}-G^{\varepsilon}\right\|_{\mathcal{L}_{2}\left(\Omega_{R}\right)}$ will be at a level $O(\varepsilon)$. Therefore, for the noise model $(5)$, there is a reason to apply a discrepancy principle in the form as proposed by SCHOCK (1983), when the procedure of the choise of $\gamma_{J}$ is terminated at the more realistic level of the residuals. Namely, $\gamma_{J}$ is chosen so that

$$
J=\min \left\{m:\left\|\Lambda_{\frac{R}{r}} F_{m}^{\varepsilon}-G^{\varepsilon}\right\|_{\mathcal{L}^{2}\left(\Omega_{R}\right)} \leq d \frac{\varepsilon^{q_{1}}}{\gamma_{m}^{q_{2}}}, \quad m=1,2, \ldots\right\}, q_{1}, q_{2}>0 .
$$

We are going to discuss this choice strategy in our forthcoming papers from mathematical point of view.

In physical geodesy one is confronted with the following situation: It is reasonable to believe that the mass density function of the earth's interior generating the earth's gravitational potential via the Newton potential is square-integrable. In consequence, a mathematical study (using the trace theorem, cf. SVENSSON (1983)) shows us that a square-integrable density inside the sphere $\Omega_{R}$ implies a potential $F_{*}$ of class $\mathcal{H}_{s}\left(\Omega_{R}\right)$ with $s=3 / 2$. Moreover, assuming a mean earth's radius of $6378[\mathrm{~km}]$ and an altitude of GOCE of about $r-R=250[\mathrm{~km}]$ we obtain $\ln ^{s}\left(\frac{r}{R}\right)^{2}=2.132 \ldots \cdot 10^{-2}$. In addition, the (unitless) leading coefficients of the earth's anomalous potential $F_{*}$ are known. This allows us to estimate $\left\|F_{*}\right\|_{3 / 2}$ via the Parseval identity

$$
\left\|F_{*}\right\|_{3 / 2}^{2}=\sum_{n=2}^{\infty} \sum_{k=1}^{2 n+1}\left(n+\frac{1}{2}\right)^{3}\left(F_{*}, Y_{n, k}^{R}\right)^{2}
$$

as follows:

$$
\left\|F^{*}\right\|_{3 / 2} \approx 1.934 \ldots \cdot 10^{-3} .
$$

Altogether this shows us that

$$
C \approx 4.125 \ldots \cdot 10^{-5}
$$


The following table gives more information about the constant $C$ for different altitudes $r-R$ :

\begin{tabular}{l|l}
$r-R[k m]$ & $\left\|F_{*}\right\|_{3 / 2} \ln ^{3 / 2}\left(\frac{r}{R}\right)^{2}$ \\
\hline 200 & $2.968 \ldots \cdot 10^{-5}$ \\
250 & $4.125 \ldots \cdot 10^{-5}$ \\
300 & $5.392 \ldots \cdot 10^{-5}$ \\
350 & $6.756 \ldots \cdot 10^{-5}$ \\
400 & $8.208 \ldots \cdot 10^{-5}$ \\
450 & $9.740 \ldots \cdot 10^{-5}$ \\
500 & $1.134 \ldots \cdot 10^{-4}$.
\end{tabular}

Choosing from Rummel, V. Gelderen (1992) (see also Bassanino et al. (1991)) $\varepsilon=10^{-11}$ (such that $\gamma_{J} \approx 10^{-6}$ ) and putting $\varepsilon$ into Theorem 3.1, where the constant $C$ takes the value $4.125 \ldots \cdot 10^{-5}$, we obtain an accuracy of about

$$
3.236 \ldots \cdot 10^{-7}
$$

for the square-root of the risk $\mathbb{E}\left\|F_{*}-F_{J}^{\varepsilon}(\xi)\right\|_{\mathcal{L}^{2}\left(\Omega_{R}\right)}^{2}$. This result is consistent with the numerical calculations presented in the PhD-thesis (by ScHNEIDER (1997)). 


\section{References}

[1] Bassanino, M., Migliaccio, F.,Sansò, F. (1991): Progress in the Spacewise Approach to ArIstoteles Data Reduction. in: ESA SP329. The Solid-Earth Mission ARIStoteles, 33-43.

[2] Baumeister, J. (1987): Stable Solution of Inverse Problems. Friedr. Vieweg \& Sohn, Braunschweig, Wiesbaden.

[3] Donoho, D.L. (1995): Nonlinear Solution of Linear Inverse Problems by Wavelet-vaguelette Decomposition. Appl. Comput. Harm. Analysis 2: $101-126$.

[4] ESA (1999): Gravity Field and Steady-State Ocean Circulation Mission (GOCE), ESA SP-1233(1) ESA Publications Division, c/o ESTEC, Nordwijk, The Netherlands.

[5] Freeden, W. (1999): Multiscale Modelling of Spaceborne Geodata, B.G. Teubner, Stuttgart, Leipzig.

[6] Freeden, W., Gervens, T., Schreiner, M. (1998): Constructive Approximation on the Sphere (With Applications to Geomathematics). Oxford University Press, Clarendon Press.

[7] Freeden, W., Schneider, F. (1998): Regularization Wavelets and Multiresolution. Inverse Problems 14: 225-243.

[8] Freeden, W., Schneider, F., Schreiner, M. (1997): Gradiometry - An Inverse Problem in Modern Satellite Geodesy, in: Inverse Problems in Geophysical Applications, 179-239, GAMM-SIAM Symposium on Inverse Problems in Geophysics, Engl, H.W., Louis, A.K., Rundell, W. eds.

[9] Golubev, G. Khasiminski R. (1997): Statistical Approach to Inverse Boundary Value Problems for Partial Differential Equations. Preprint, No. 370. WIAS-Berlin.

[10] ILK, K.H. (1993): Regularization for High Resolution Gravity Field Recovery by Future Satellite Techniques. Inverse Problems: Principles and Applications in Geophysics, Technology and Medicine, Anger, G. et al. (eds.), Math. Research, Vol. 74, Akademie Verlag, 189-214.

[11] JeKeli, C. (1988): The Gravity Gradiometer Survey System (GGSS), EOS, Trans. Amer. Geophys. Un. 69: 105-117. 
[12] Korostelev, A.P., Tsybakov, A.B. (1993): Minimax Theory of Image Reconstruction. Lecture Notes Statist. 82. Springer, New York.

[13] Kusche, J. (1998): Regional adaptive Schwerefeldmodellierung für SST-Analysen. In: Progress in Geodetic Science at GW98, (W. Freeden ed.), Shaker, 266-273.

[14] Louis, A.K. (1982): Inverse und schlecht gestellte Probleme, B.G. Teubner, Stuttgart.

[15] MAIR, B.A. (1994): Tikhonov Regularization for Finitely and Infinitely Smoothing Operators. SIAM J. Math. Anal., 25: 135-147.

[16] Mair, B.A., Ruymgaart, F.M. (1996): Statistical Inverse Estimation in Hilbert Scales. SIAM J. Appl. Math. 56: 1424-1444.

[17] Nussbaum, M. (1994): Degrees of Ill-posedness in Stochastic and Deterministic Noise Models. Dagstuhl-Seminar-Report 101.

[18] Pereverzev, S., Schock, E. (1999): Error Estimates for Bandlimited Spherical Regularization Wavelets in an Inverse Problem of Satellite Geodesy. Inverse Problems 15: 881-890.

[19] Pereverzev, S., Schock, E. (2000): Morozov's Discrepancy Principle for Tikhonov Regularization of Severely Ill-posed Problems in Finite-dimensional Subspaces. Preprint No. 318, Fachbereich Mathematik, Universiät Kaiserslautern.

[20] Rummel, R. (1997): Spherical Spectral Properties on the Earth's Gravitational Potential and Its First and Second Derivatives, in: Lecture Notes in Earth Sciences 65, 359-405, Springer, Heidelberg.

[21] Rummel, R., V. Gelderen, M. (1992): Spectral Analysis of the Full Gravity Tensor. Geophy. J. Int., 111: 159-169.

[22] Rummel, R., V. Gelderen, M., Koop, R., Schrama, E., Sansò, F., Brovelli, M., Miggliaccio, F., Sacerdote, F. (1993): Spherical Harmonic Analysis of Satellite Gradiometry. Netherlands Geodetic Commission, New Series, No. 39.

[23] SchneIder, F. (1997): Inverse Problems in Satellite Geodesy and their Approximate Solution by Splines and Wavelets. Doctoral Thesis, University of Kaiserslautern, Geomathematics Group, Shaker, Aachen. 
[24] Schock, E. (1984): Parameter Choice by Discrepancy Principle for the Approximate Solution of Ill-posed Problems. Integral Equations and Operator Theory, 7: 895-898.

[25] Svensson, S.L. (1983): Peudodifferential Operators - a New Approch to the Boundary Value Problems of Physical Geodesy. Manuscr. Geod., 8: $1-40$.

[26] Tscherning, C.C., Forsberg, R., Vermeer, M. (1990): Methods for Regional Gravity Field Modelling from SST and SGG. Reports of the Finnish Geodetic Institute 90:2.

[27] Wahba, G. (1977): Practical Approximate Solutions to Linear Operator Equations When the Data are Noisy. SIAM J. Numer. Anal. 14:651-667. 